\title{
Agent Action Map Oriented Intelligent Simulation System for Emergency Management
}

\author{
Xiong $\mathrm{Li}^{\mathrm{a}}$, Wei $\mathrm{Pu}^{\mathrm{b}}$ \\ Department of Command and Administration \\ Academy of Armored Force Engineering, 100072, Beijing \\ alixiong2609@126.com, byyzhxlw@126.com
}

Keywords: Intelligent Simulation System, Agent, Agent-based Simulation, Agent Action Map, Emergency Management.

Abstract. The purpose of this paper is to explore agent action map oriented intelligent simulation system model used in emergency management. This paper establishes the concept of agent action map and thus designs agent action map oriented intelligent simulation system. It further presents a method in transforming real entities and system to organization map model, capability attributes map model, single-agent action map model and multi-agent action map model. The application in fateful traffic accident management simulation proves that this approach is feasible and effective.

\section{Introduction}

With the evolution of technology, the methods for communicating emergency situations have also changed, as has the definition for what might constitute an "emergency". These methods would also very likely depend on particular public crisis situations. Thus, emergency management is being an important decision-making action, using some intelligent simulation tools with the ability to provide specific instructions on what to do in order to mitigate the effects of an emergency.

The development of intelligent simulation system technology has also changed the way we think about emergency management. Public crisis, e.g., a fateful traffic accident is usually distributed, in which updates are made asynchronously and resources come online and go offline without centralized control. In order to demonstrate these resources dynamically in a computer system, without public crisis risks, we need a reasonable intelligent simulation system [1].

Agent-based simulation paradigm has become a useful tool in analyzing complex process and system $[2,3,4,5,6,7,8]$. Thus, it can be viewed as a feasible method to explore emergency management. However, there are a lot of difficulties to take some factors such as autonomy, reactivity, sociality, adaptability and intelligence into account. Conventional agent-based simulation system needs be adjusted and improved to meet the requirements of intelligent decision-making in emergency management. In this paper, we design an agent action map oriented intelligent simulation system model to demonstrate some emergent activities during dealing with public crisis.

\section{From Agent to Agent Action Map}

Agent and multi-agent system emerged, as a scientific area, from the previous research efforts in distributed artificial intelligence started in 1980s. The analysis and comprehensive understanding of intelligent systems is extremely difficult and in most cases impossible for humans to grasp without the assistance of advanced tool such as agent-based simulation. Agent-based simulation technology gives concept developers a view to the future by enabling them to simulate and manipulate in near real-time, the assets and operational conditions. In recent years, agent-based simulation approaches to intelligent systems have gained increasing attention.

On the one hand, agents have intentions and actions. They are autonomous, flexible and proactive. In addition, agents have multi-threaded control and can build a control to act only if they want to. An agent is a system that is capable of perceiving events in its environment, or representing information about the current state of affairs and of acting in its environment guided by perceptions and stored 
information. In a multi-agent system there are a lot of interaction activities such as cooperation (working together to achieve a common objective), coordination (arranging inter-dependent activities) and negotiation (coming to a mutually acceptable agreement on issues) $[2,3,4,5,6,7,8]$. On the other hand, the internal members of emergency management system run with autonomy and interaction. These information interactions include sending, accepting, and informing all kinds of command and control instruction, navigation information, imagery intelligence, signals intelligence, measurement intelligence, and surveillance information. Emergency management system is so alike a distributed multi-agent system in behaviors that we can set up mappings from its internal members, i.e., emergency management entities, to respective agents, e.g., transportation section $\rightarrow$ transportation section agent, and police command vehicle $\rightarrow$ police command vehicle agent.

In order to explore syllabify the above interaction actions in emergency management system, we propose the concept of agent action map and thus design agent action map oriented intelligent simulation system. In an agent action map, the organization of multi-agent system and capability attributes of an entity agent are shown as certain figures. Accordingly, agent action map not only represents the emergency management entity actions, but also represents the system organization actions.

The key to design agent action map model is analisis of emergency management system including organization and entities. The emergency management system organization - action - entity - agent mapping relationship mechanism shown as Fig. 1 [1] is helpful to transform real emergency management entities and system to intelligent model.

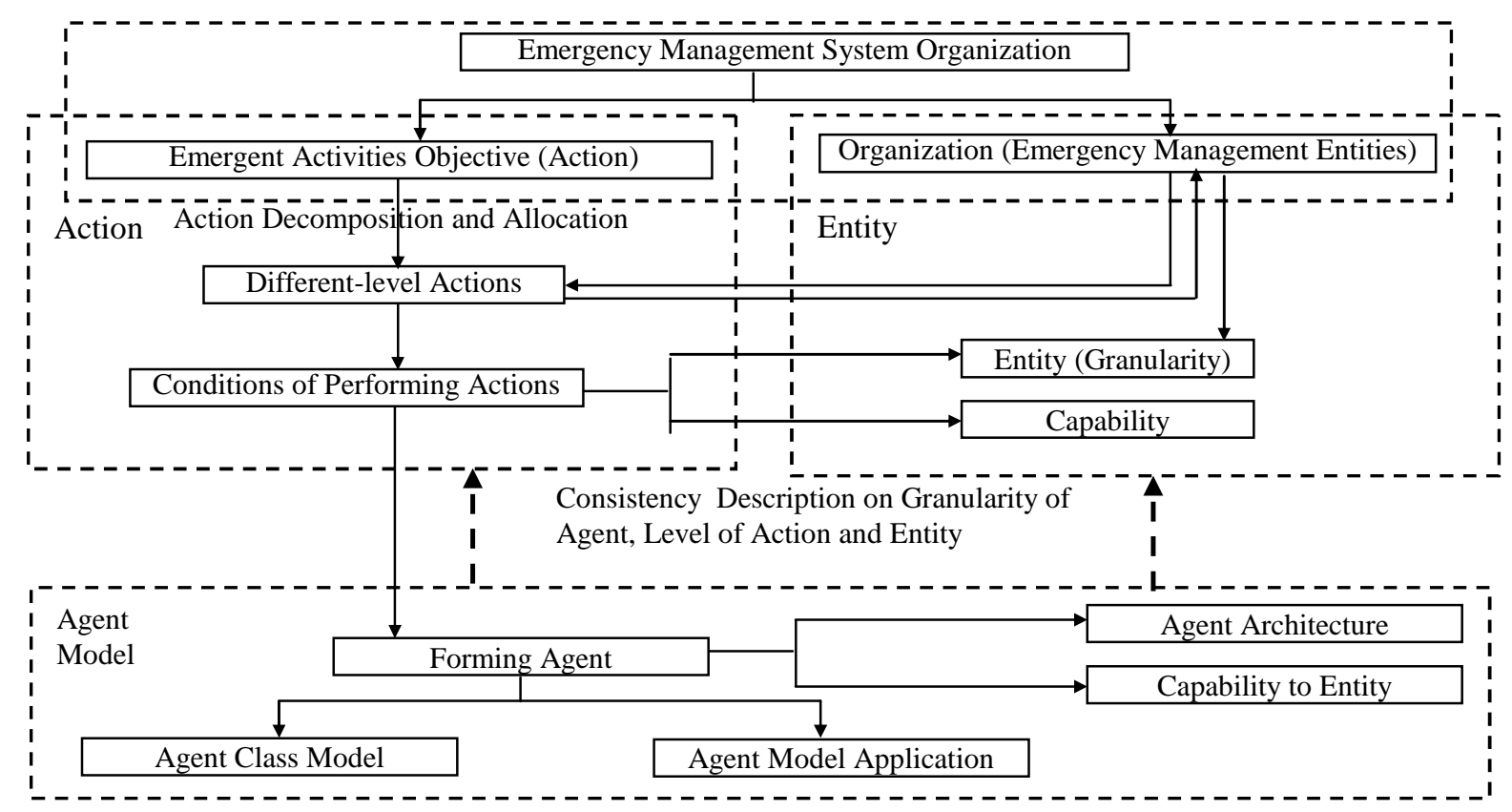

Fig.1 Mapping relationship mechanism.

\section{Intelligent Simulation System Design and Case Study}

In this paper, we take a fateful traffic accident as an example of public crisis. Thus, we study emergency management for this fateful traffic accident, and accordingly observe the microcosmic entity actions and organizational system actions.

The intelligent simulation system that we set up can be illustrated by Fig. 2. Fig. 2 presents the dynamic and real-time situation information during agent action map oriented intelligent simulation. By this system, one can find out easily a certain emergency management entity agent's real-time state information. 


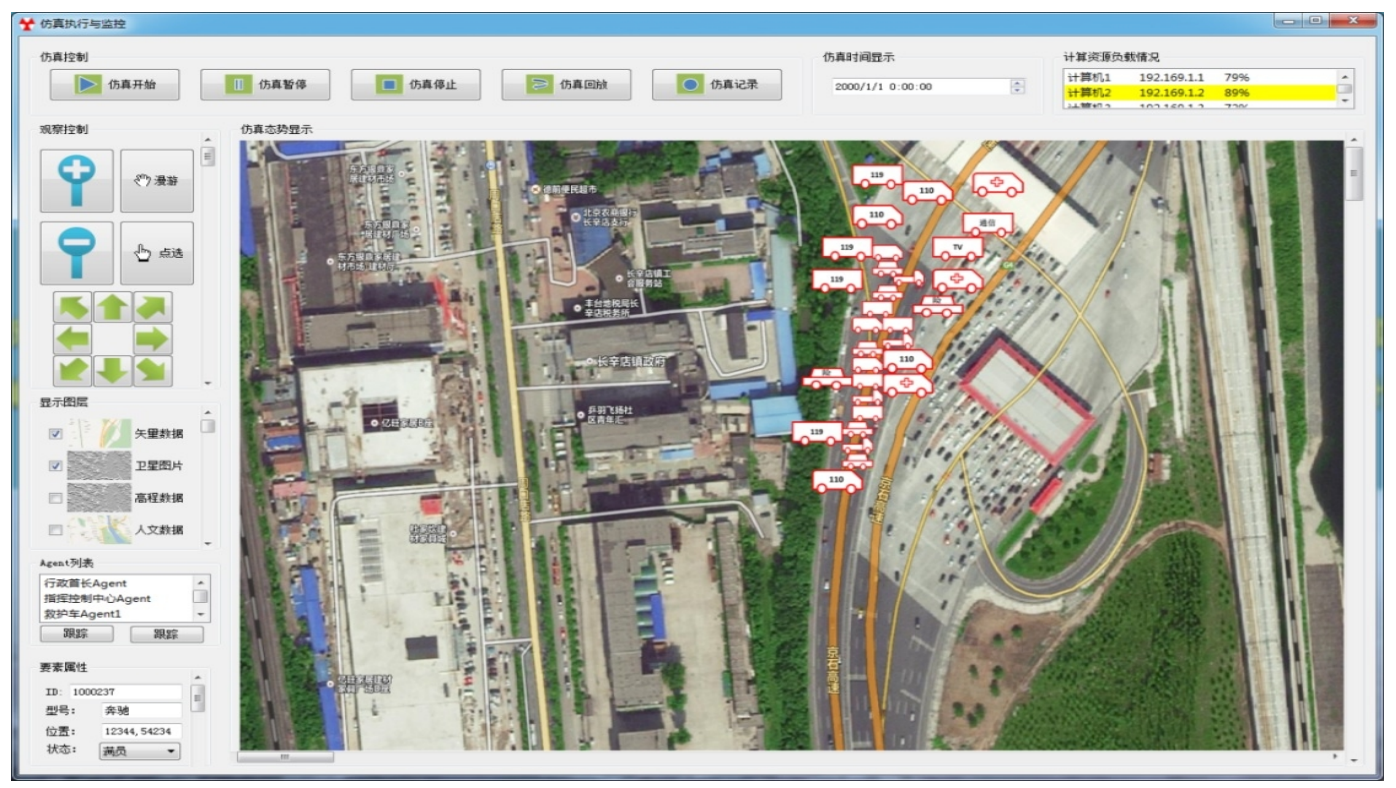

Fig.2 Intelligent simulation system.

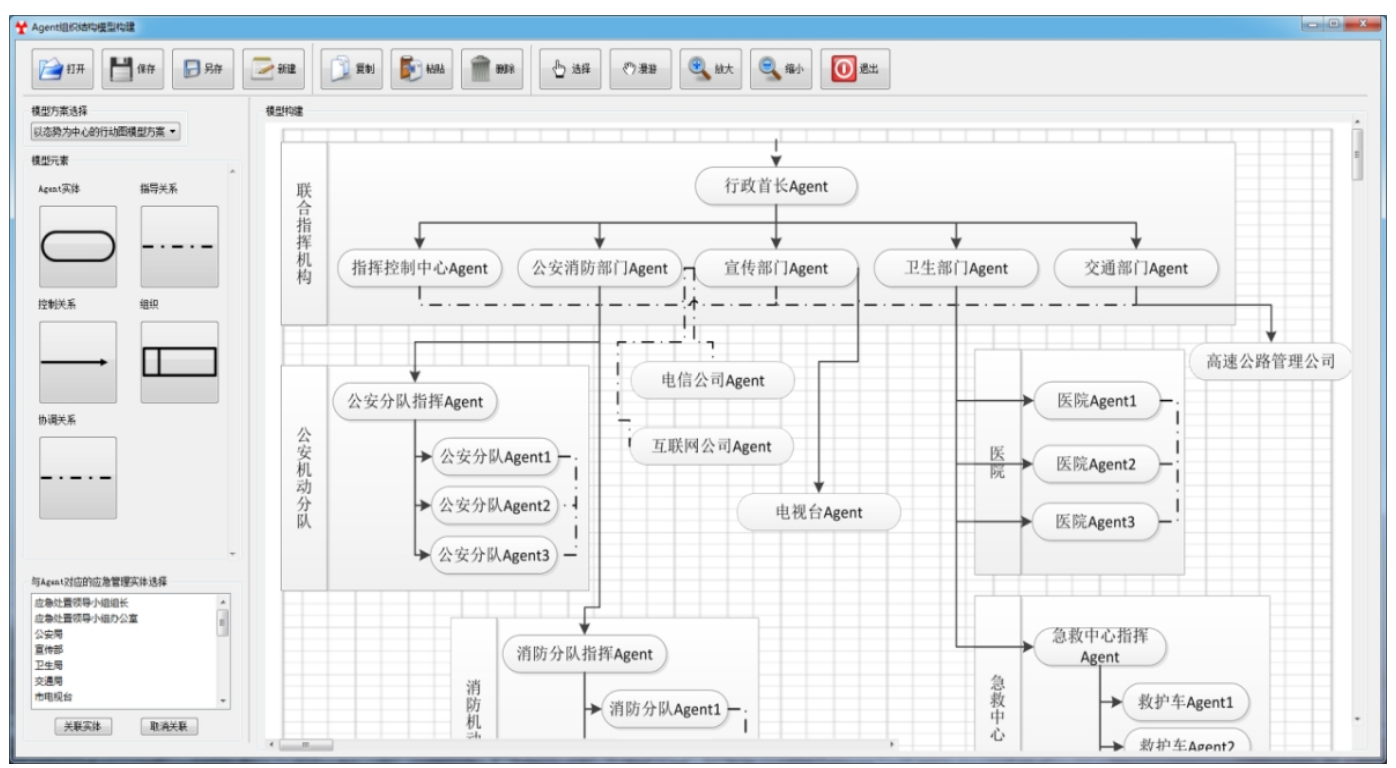

Fig. 3 Organization map model design.

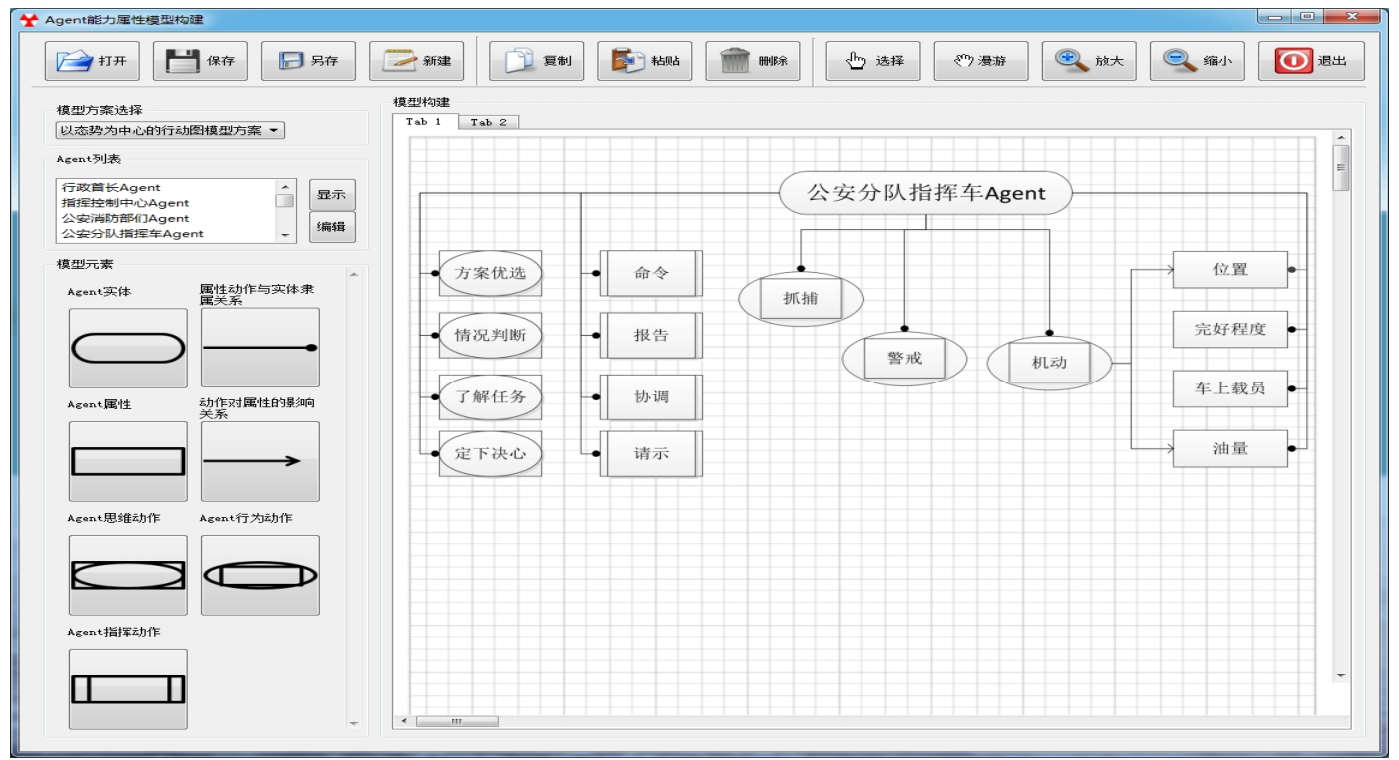

Fig. 4 Capability attributes map model design. 


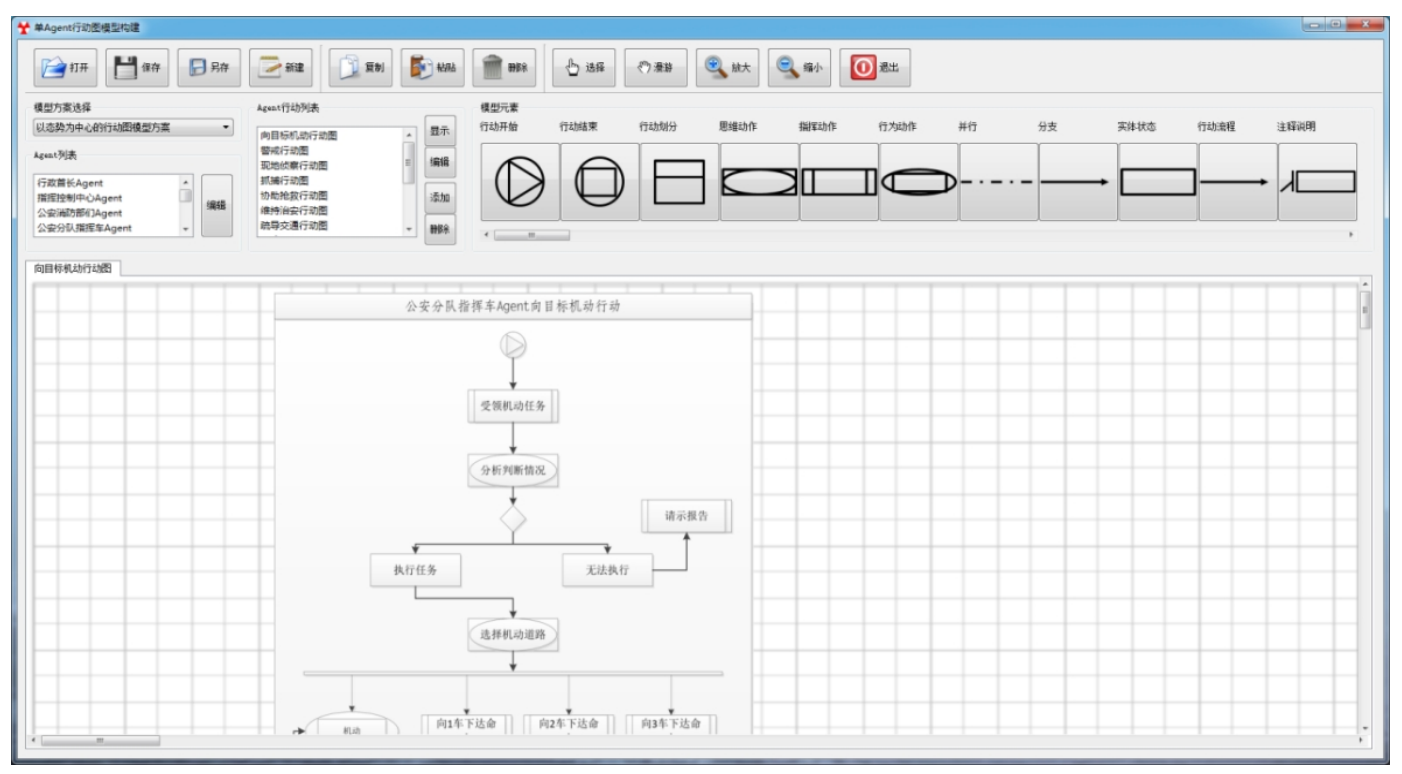

Fig. 5 Single-agent action map model design.

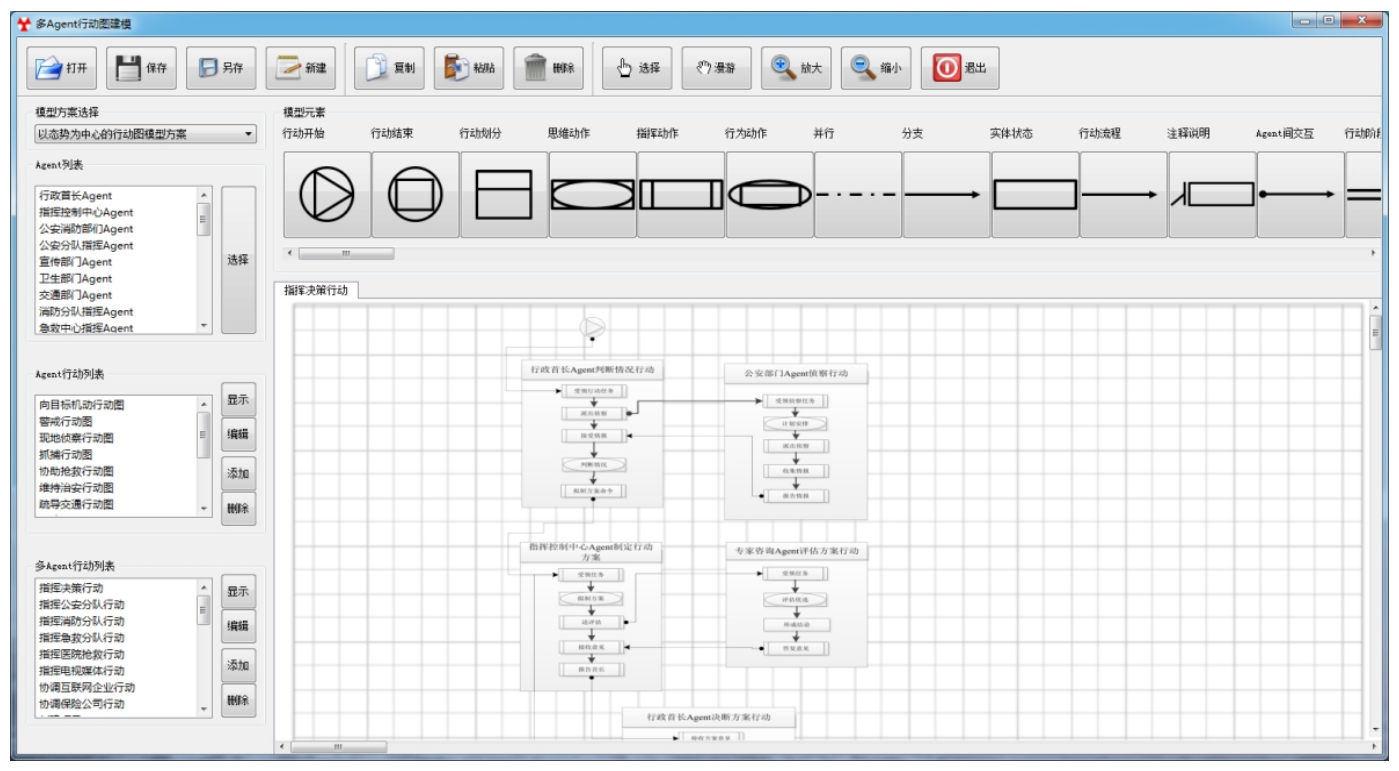

Fig. 6 Multi-agent action map model design.

In the intelligent simulation system, organization map model design, capability attributes map model design, single-agent action map model design and multi-agent action map model design can be respectively illustrated by Fig. $3 \sim$ Fig. 6 .

In this case study, 100 times simulations were implemented, and results of situation-centric action scheme and plan-centric action scheme were contrasted, illustrated by Tab. 1. According to the results, one can find out easily that situation-centric action scheme is better than plan-centric action scheme. This fact also shows that situation-centric emergency management play an important role in dealing with public crisis.

Tab. 1 Simulation results of two action schemes.

\begin{tabular}{|c|c|c|}
\hline Index & Situation-centric Action Scheme & Plan-centric Action Scheme \\
\hline Mean time to cure for injured ones & $30.12 \mathrm{~min}$ & $45.98 \mathrm{~min}$ \\
\hline Mean time of police arriving & $15.03 \mathrm{~min}$ & $25.10 \mathrm{~min}$ \\
\hline Mean time of firemen arriving & $20.96 \mathrm{~min}$ & $35.28 \mathrm{~min}$ \\
\hline Mean time of command and decision-making & $5.09 \mathrm{~min}$ & $9.19 \mathrm{~min}$ \\
\hline Mean time of measuring vehicle damage & $75.12 \mathrm{~min}$ & $98.92 \mathrm{~min}$ \\
\hline Mean time of medium arriving & $28.97 \mathrm{~min}$ & $49.39 \mathrm{~min}$ \\
\hline
\end{tabular}


We carry through Verification, Validation, and Accreditation (VV\&A) for our agent action map oriented intelligent simulation system model to analyze these results. As far as the concept model, we check whether attributes description and interactions are consistent with real public crisis situation. As far as the program model, emphases are put in data to verify their correctness, dependability and performance [2].

By the evaluation, these results that we obtained from agent action map oriented intelligent simulation are accordant to real public crisis situation. The fact proves that our model is feasible and effectual.

\section{Conclusions}

For some time now agent-based simulation for intelligent systems has attracted the interest of researchers far beyond traditional computer science. According to the demands of modeling and simulation for emergent activities for public crisis, agent action map oriented intelligent simulation system model is presented in this paper. The key step in this approach is transforming real emergency management entities and emergency management system to organization map model, capability attributes map model, single-agent action map model and multi-agent action map model. The agent action map oriented intelligent simulation system model can establish a bridge to connect prototype and simulation, thus software engineers can design model and implement intelligent simulation system with more convenience.

\section{Acknowledgements}

This work is supported by the National Natural Science Foundation of China under Grant 61473311, Beijing Natural Science Foundation under Grant 9142017 and Scientific Research Projects of Chinese PLA.

\section{References}

[1] Xiong Li, Wei Pu. Task Oriented Equipment-level Agent-based Modeling for Emergency Operations. Proceedings of the International Conference on Materials Processing and Mechanical Manufacturing Engineering, Changsha, China, September 19-20, 2015, DEStech Publications. p. 47-52.

[2] Xiong Li, Zhiming Dong. Platform-Level Distributed Warfare Model Based on Multi-Agent System Framework, Defence Science Journal, Vol. 62(3) (2012), p. 180-186.

[3] Xiong Li. Agent-based Warfare Modeling (National Defense Industry Press, Beijing, China 2013).

[4] Zhongzhi Shi. Intelligent Agents and Their Applications (Science Press, Beijing, China 2000).

[5] Zarboutis N., Marmaras N. Design of Formative Evacuation Plans Using Agent-based Simulation, Safety Science, Vol. 45(9) (2007), p. 920-940.

[6] Victor Lesser. Autonomous Agents and Multi-Agent Systems (Kluwer, Boston, USA 1998).

[7] J. Ferber. Multi-Agent Systems: An Introduction to Distributed Artificial Intelligence (Addison-Wesley, 1999).

[8] Liu Jinfei, Chen Ming, Wang Lei, Wu Qidi. A Task-oriented Modular and Agent-based Collaborative Design Mechanism for Distributed Product Development, Chinese Journal of Mechanical Engineering, Vol. 27(3) (2014), p. 641-654. 